\title{
Modeling of Microstructure Changes in FePt Nano-Granular Thin Films Using the Phase-Field Method
}

\author{
Toshiyuki Koyama* and Hidehiro Onodera \\ Computational Materials Science Center, Independent Administrative Institution National Institute for Materials Science, \\ Tsukuba 305-0047, Japan
}

\begin{abstract}
Since the factors that influence microstructure formation are extensive (e.g., alloy composition, heat treatment condition, etc.), quite a lot of experimental trial-and-error is often necessary when searching for the best combination of desired microstructure and material properties, even when the basic mechanism of microstructure formation is understood. During the last decade, the phase-field method has emerged across many fields in materials science as a powerful tool to simulate and predict complex microstructure evolution. Since phase-field methodology can model complex microstructure changes quantitatively, it is possible to search for the most desirable microstructure by using this method as a design simulation, i.e., through computer trial-and-error testing. In order to establish this methodology, first of all, quantitative modeling of complex microstructure changes using the phase-field method is required. The objective of this study is to model the FePt nano-granular structure formation and the order-disorder phase transition of FePt nano-particles as a typical example of phase-field modeling. We show that it is possible to model FePt nano-granular structure formation quantitatively using the phase-field method. This modeling method may also be applicable to various types of granular structure formation. The simulation result also suggests that there is a size dependence on the ordering of FePt nano-particles. Using the phase-field method to model the microstructure evolutions proved to be a very effective strategy in predicting and analyzing the complex microstructure formation.
\end{abstract}

(Received March 20, 2003; Accepted April 28, 2003)

Keywords: phase-field method, phase transformation, phase decomposition, pattern formation, complex system, diffusion equation, evolution equation, microstructure, energy, non-linear

\section{Introduction}

Since the factors that influence microstructure formation are extensive (e.g., alloy composition, heat treatment condition, etc.), quite a lot of experimental trial-and-error is often necessary when searching for the best combination of desired microstructure and material properties, even when the basic mechanism of microstructure formation is understood. During the last decade, the phase-field method ${ }^{1-5)}$ has emerged across many fields in materials science as a powerful tool to simulate and predict complex microstructure evolution, (e.g., dendrite growth, spinodal decomposition, Ostwald ripening, crystal growth, recrystallization, martensitic transformation, dislocation dynamics, electromigration, crack propagation, and so on). Since phase-field methodology can model complex microstructural changes quantitatively, it is possible to search for the most desirable microstructure by using this method as a design simulation, i.e., through computer trial-and-error testing. Therefore, the most effective strategy for developing advanced materials is as follows: First, we elucidate the mechanism of microstructure changes experimentally, and then modeling the microstructure evolutions using the phase-field method based on the experimental results, and finally we search for the most desirable microstructure while simultaneously considering both the simulation and experimental data. In order to carry out this methodology, the flexible quantitative modeling method for complex microstructure changes using the phase-field method must first be established. For simplicity, "modeling of microstructure changes using the phase-field method" will be referred to as "phase-field modeling" throughout the rest of this paper. Phase-field modeling, from a practical viewpoint, is aimed at decreasing the frequency of experimental trial-and-error testing as much as possible through modeling and computer simulations.

In this study, as a typical example of phase-field modeling, we propose a model for FePt nano-granular microstructure formation during sputtering and the order-disorder phase transition of FePt nano-particles in nano-granular thin film during subsequent annealing. ${ }^{6,7)}$ The FePt nano-granular structure is considered to be a candidate for the next generation of high-density recording media due to its large magneto-crystalline anisotropy.

The phase-field method has been developed extensively in the field of materials science, and has come to be used in two different ways. One is the phase-field method used for simulation techniques on interface dynamics, e.g., where solidification process occurs, and the other is the continuum field model describing order parameters for temporal and spatial development during phase transformations. In this study, the latter definition of phase-field is used. We propose a modeling method for microstructure changes in FePt nanogranular thin films based on phase-field modeling.

\section{Phase-Field Method}

The phase-field method is explained below. Details of this method will be obtained from Refs. 1)-5). In general, the phase-field method is a computer simulation method for calculating the dynamics of temporal microstructure changes by solving the continuum non-linear evolution equations defined by eqs. (1) and (2). These equations include the dynamic coupling terms for the order parameters of time variation. 


$$
\begin{aligned}
\frac{\partial c_{i}(\boldsymbol{r}, t)}{\partial t}= & \nabla \cdot\left\{M_{c_{i}}\left\{c_{i}(\boldsymbol{r}, t), T\right\}\left[\nabla \frac{\delta G_{\mathrm{sys}}}{\delta c_{i}(\boldsymbol{r}, t)}+\xi_{c_{i}}(\boldsymbol{r}, T, t)\right]\right\} \\
& +K_{c}\left\{c_{i}(\boldsymbol{r}, t), s_{j}(\boldsymbol{r}, t), T\right\} \frac{\partial s_{j}(\boldsymbol{r}, t)}{\partial t} \\
\frac{\partial s_{j}(\boldsymbol{r}, t)}{\partial t}= & -L_{s_{j}}\left\{s_{j}(\boldsymbol{r}, t), T\right\}\left[\frac{\delta G_{\mathrm{sys}}}{\delta s_{j}(\boldsymbol{r}, t)}+\xi_{s_{j}}(\boldsymbol{r}, T, t)\right] \\
& +K_{s}\left\{c_{i}(\boldsymbol{r}, t), s_{j}(\boldsymbol{r}, t), T\right\} \frac{\partial c_{i}(\boldsymbol{r}, t)}{\partial t}
\end{aligned}
$$

$T$ is absolute temperature. $c_{i}(\boldsymbol{r}, t)$ and $s_{j}(\boldsymbol{r}, t)$ are the order parameters for the conserved and non-conserved fields, respectively, and these are a function of position $\boldsymbol{r}$ and time $t$. Subscript $i$ and $j$ for each order parameter distinguish order parameters belonging to the same category. The order parameters such as $c_{i}(\boldsymbol{r}, t)$ and $s_{j}(\boldsymbol{r}, t)$ are commonly called phase-field variables in the phase-field method. $M_{c_{i}}\left\{c_{i}(\boldsymbol{r}, t), T\right\}$ and $L_{s_{j}}\left\{s_{j}(\boldsymbol{r}, t), T\right\}$ are the mobility terms for microstructure changes with respect to each order parameter, and these are usually described as a function of the order parameters, but in many actual calculations, the mobility is often set to a constant or to a function of temperature. In this study, the mobility is assumed to be constant, $M_{c_{i}}=1$ and $L_{s_{j}}=1$, so that the time dimension in this calculation is normalized. $\xi_{p}(\boldsymbol{r}, t)$ is a thermal Gaussian noise term for the order parameter $p\left(=c_{i}\right.$ or $\left.s_{j}\right)$. The second term of eqs. (1) and (2) refers to the dynamic coupling of each phase-field variable, where $K_{c}\left\{c_{i}(\boldsymbol{r}, t), s_{j}(\boldsymbol{r}, t), T\right\} \quad$ and $K_{s}\left\{c_{i}(\boldsymbol{r}, t), s_{j}(\boldsymbol{r}, t), T\right\}$ are the coupling coefficients. These are often assumed to a constant, or set to 0 when dynamic feedback between order parameters is ignored. In this simulation, we don't consider these dynamical feedback terms. This coupling term should be introduced only when a highly complex microstructural morphology is formed considerably far from equilibrium, e.g., dendrite growth, fractal pattern formation, etc.

$G_{\text {sys }}$ is the total free energy of the microstructure, which is defined by the sum of the chemical free energy $G_{c}$, the interfacial energy $E_{\text {surf }}$, and the elastic strain energy $E_{\text {str }}$, so that the functional $G_{\text {sys }}$ is written as:

$$
G_{\mathrm{sys}}=\int_{\boldsymbol{r}}\left[\begin{array}{l}
G_{c}\left\{c_{i}(\boldsymbol{r}, t), s_{j}(\boldsymbol{r}, t), T\right\}+E_{\mathrm{surf}}\left\{c_{i}(\boldsymbol{r}, t), s_{j}(\boldsymbol{r}, t), T\right\} \\
+E_{\mathrm{str}}\left\{c_{i}(\boldsymbol{r}, t), s_{j}(\boldsymbol{r}, t), T\right\}
\end{array}\right] d \boldsymbol{r} .
$$

Since $G_{c}, E_{\text {surf }}$, and $E_{\text {str }}$ are expressed as a function of order parameters, $c_{i}(\boldsymbol{r}, t)$ and $s_{j}(\boldsymbol{r}, t)$, these parameters interact with each other through the total free energy during microstructure changes in the computer simulation. Moreover, these variables interact dynamically when the dynamic coupling term is considered.

\section{Modeling of Microstructure Changes in FePt Nano- Granular Thin Films}

\subsection{Formulation of total free energy}

The desired microstructure change for the phase-field modeling in this study is to have the FePt nano-granular structure where the FePt nano-particles are distributed in a$\mathrm{Al}_{2} \mathrm{O}_{3}$ (amorphous alumina), produced by sputtering process. According to experimental results obtained by Watanabe ${ }^{6}$ and Ping et al. ${ }^{7)}$ the features of FePt nano-granular structure are as follows:

(1) Three phases have been identified experimentally, i.e., the a- $\mathrm{Al}_{2} \mathrm{O}_{3}$ phase, disordered FePt phase (A1), and ordered FePt phase $\left(\mathrm{L}_{0}\right)$.

(2) Since the as-sputtered FePt nano-granular structure shows the morphology of a two-phase mixture containing a- $\mathrm{Al}_{2} \mathrm{O}_{3}$ and the disordered FePt (A1), the phase separated state, i.e., $\mathrm{a}-\mathrm{Al}_{2} \mathrm{O}_{3}$ phase $+\mathrm{FePt}$ phase is more stable than forming a solid solution.

(3) The FePt phase is in its disordered state (A1) in the assputtered film, and order-disorder phase transition from A1 to $\mathrm{L}_{0}$ takes place in the FePt phase during subsequent thermal treatment.

In this study, we attempt to model these microstructure changes using phase-field modeling. Since phase-field modeling deals with the design of real microstructure changes quantitatively, several physically unclear assumptions have occasionally been adopted. Although there may be several insufficient parts in this model, for practical engineering applications, we believe this methodology will be useful for actual materials research and development.

In order to describe temporal changes of microstructure, the order parameter $\phi_{\mathrm{FePt}}(\boldsymbol{r}, t)\left(0 \leq \phi_{\mathrm{FePt}} \leq 1\right)$, the probability of finding the FePt phase at time $t$ and position $\boldsymbol{r}$, and the degree of order $s(\boldsymbol{r}, t)$ of the $\mathrm{FePt}\left(\mathrm{L} 1_{0}\right)$ phase are employed as phase-field variables. The value $s(\boldsymbol{r}, t)$ is normalized by the maximum equilibrium value of degree of order determined by the FePt composition and temperature, so that the range of $s(\boldsymbol{r}, t)$ is set between $(0 \leq|s| \leq 1)$. According to the initial assputtered stage experimental evidence, some amount of $\mathrm{Fe}$ and $\mathrm{Pt}$ elements are dissolved in the $\mathrm{a}-\mathrm{Al}_{2} \mathrm{O}_{3}$ phase, and the volume fraction of $\mathrm{FePt}$ phase increases during aging. Therefore the concentrations of $\mathrm{Fe}$ and $\mathrm{Pt}$ can also be employed as order parameters. However, to make the present simulation model simpler we assume that all of the Fe and Pt atoms are contained in the FePt phase and the composition of FePt particles is constant.

The total free energy function is evaluated by utilizing the defined order parameters. Thermodynamic data which obtained from a thermodynamic database of $\mathrm{Fe}-\mathrm{Pt}$ phase diagram is the Gibbs energies of disordered FePt phase (A1), $G_{\mathrm{FePt}}^{\mathrm{A} 1}\left(c_{\mathrm{Fe}}, c_{\mathrm{Pt}}, T\right)$, and ordered FePt phase $\left(\mathrm{L}_{0}\right)$, $G_{\mathrm{FePt}}^{\mathrm{L} 1_{0}}\left(c_{\mathrm{Fe}}, c_{\mathrm{Pt}}, T\right)$, where $c_{i}$ is the composition of element $i$. The chemical free energy functions for the " $\mathrm{a}-\mathrm{Al}_{2} \mathrm{O}_{3}$ $\mathrm{FePt}(\mathrm{A} 1)$ system" and the "a- $\mathrm{Al}_{2} \mathrm{O}_{3}-\mathrm{FePt}\left(\mathrm{L}_{0}\right)$ system" are given respectively as: 


$$
\begin{aligned}
G_{\mathrm{Al}_{2} \mathrm{O}_{3}-\mathrm{A} 1} & =\left(1-\phi_{\mathrm{FePt}}\right) G_{\mathrm{Al}_{2} \mathrm{O}_{3}}^{\mathrm{am} 1}(T)+\phi_{\mathrm{FePt}} G_{\mathrm{FePt}}^{\mathrm{Al}}\left(c_{\mathrm{Fe}}, c_{\mathrm{Pt}}, T\right)+W_{\mathrm{Al}_{2} \mathrm{O}_{3}-\mathrm{A} 1} g^{2}\left(\phi_{\mathrm{FePt}}\right) \\
G_{\mathrm{Al}_{2} \mathrm{O}_{3}-\mathrm{L} 1_{0}} & =\left(1-\phi_{\mathrm{FePt}}\right) G_{\mathrm{Al}_{2} \mathrm{O}_{3}}^{\mathrm{am} 2}(T)+\phi_{\mathrm{FePt}} G_{\mathrm{FePt}}^{\mathrm{L} 1_{0}}\left(c_{\mathrm{Fe}}, c_{\mathrm{Pt}}, T\right)+W_{\mathrm{Al}_{2} \mathrm{O}_{3}-\mathrm{L}_{0}{ }_{0}} g^{2}\left(\phi_{\mathrm{FePt}}\right),
\end{aligned}
$$

where the function $g(\phi)$ is defined by $g(\phi) \equiv \phi(1-\phi){ }^{1,4)} G_{\mathrm{Al}_{2} \mathrm{O}_{3}}^{\mathrm{am}}(T)$ and $G_{\mathrm{Al}_{2} \mathrm{O}_{3}}^{\mathrm{am} 2}(T)$ are the Gibbs energy of the a- $\mathrm{Al}_{2} \mathrm{O}_{3}$ phase before and after the ordering of the FePt phase, respectively. $W_{\mathrm{Al}_{2} \mathrm{O}_{3}-\mathrm{A} 1}$ and $W_{\mathrm{Al}_{2} \mathrm{O}_{3}-\mathrm{L}_{0}}$ are the energy barriers terms between the a- $\mathrm{Al}_{2} \mathrm{O}_{3}$ and the FePt phase. These terms ensure that the phase separation for these two phases is stable, and we assume $W_{\mathrm{Al}_{2} \mathrm{O}_{3}-\mathrm{Al}}=W_{\mathrm{Al}_{2} \mathrm{O}_{3}-\mathrm{L}_{0}}$. By using eqs. (4) and (5), the chemical free energy of the system is expressed as:

$$
G_{c}=\{1-h(s)\} G_{\mathrm{Al}_{2} \mathrm{O}_{3}-\mathrm{A} 1}+h(s) G_{\mathrm{Al}_{2} \mathrm{O}_{3}-\mathrm{L} 1_{0}}+W_{{\mathrm{A} 1-\mathrm{L}_{0}}_{0}} g^{2}(s),
$$

where $s$ is the $\mathrm{L}_{0}$ ordering parameter, and the function $h(s)$ is defined by $h(s) \equiv s^{2}\left(3-2 s^{2} /|s|\right)$. ${ }^{1,4)} W_{\mathrm{A} 1-\mathrm{L} 1_{0}}$ is the energy barrier term for the order-disorder phase transition, and we assume $W_{\mathrm{A} 1-\mathrm{L}_{0}}=0$. In order to make the equations simpler, the standard reference energy level, i.e., the segregation limit of the chemical free energy, is shifted to $\left(1-\phi_{\mathrm{FePt}}\right) G_{\mathrm{Al}_{2} \mathrm{O}_{3}}^{\mathrm{am}}(T)+$ $\phi_{\mathrm{FePt}} G_{\mathrm{FePt}}^{\mathrm{A} 1}\left(c_{\mathrm{Fe}}, C_{\mathrm{Pt}}, T\right)$. Therefore, eqs. (4)-(6) reduce to:

$$
\begin{aligned}
& \Delta G_{\mathrm{Al}_{2} \mathrm{O}_{3}-\mathrm{A} 1}\left(\phi_{\mathrm{FePt}}\right)=W_{\mathrm{Al}_{2} \mathrm{O}_{3}-\mathrm{A} 1} g^{2}\left(\phi_{\mathrm{FePt}}\right) \\
& \Delta G_{\mathrm{Al}_{2} \mathrm{O}_{3}-\mathrm{L} 1_{0}}\left(\phi_{\mathrm{FePt}}\right)=\left(1-\phi_{\mathrm{FePt}}\right) \Delta G_{\mathrm{Al}_{2} \mathrm{O}_{3}}^{\mathrm{am} 1 \rightarrow \mathrm{am} 2}(T)+\phi_{\mathrm{FePt}} \Delta G_{\mathrm{FePt}}^{\mathrm{A} 1 \rightarrow \mathrm{L}_{0}}\left(c_{\mathrm{Fe}}, c_{\mathrm{Pt}}, T\right)+W_{\mathrm{Al}_{2} \mathrm{O}_{3}-\mathrm{L} 1_{0}} g^{2}\left(\phi_{\mathrm{FePt}}\right) \\
& \Delta G_{c}\left(\phi_{\mathrm{FePt}}, s\right)=\{1-h(s)\} \Delta G_{\mathrm{Al}_{2} \mathrm{O}_{3}-\mathrm{A} 1}+h(s) \Delta G_{\mathrm{Al}_{2} \mathrm{O}_{3}-\mathrm{L}_{0}}+W_{\mathrm{A} 1-\mathrm{L}_{0}} g^{2}(s),
\end{aligned}
$$

where $\Delta G_{\mathrm{Al}_{2} \mathrm{O}_{3}}^{\mathrm{am} 1 \rightarrow \mathrm{am} 2} \equiv G_{\mathrm{Al}_{2} \mathrm{O}_{3}}^{\mathrm{am} 2}-G_{\mathrm{Al}_{2} \mathrm{O}_{3}}^{\mathrm{am} 1} \quad$ and $\quad \Delta G_{\mathrm{FePt}}^{\mathrm{A1} \rightarrow \mathrm{L}_{0}} \equiv$ $G_{\mathrm{FePt}}^{\mathrm{L} 1_{0}}-G_{\mathrm{FePt}}^{\mathrm{Al}} \cdot$

The gradient energy ${ }^{1-5)}$ at the interface between the a$\mathrm{Al}_{2} \mathrm{O}_{3}$ and $\mathrm{FePt}$ phases is calculated by

$$
E_{\text {surf }}=\kappa_{\phi}\left(\nabla \phi_{\mathrm{FePt}}\right)^{2}+\kappa_{s}|\nabla s|^{2},
$$

where the gradient energy coefficients $\kappa_{\phi}$ and $\kappa_{s}$ are constants. The elastic strain energy is assumed to be 0 , because, in the present case, the elastic constraint can be ignored.

In the next section, we will explain how to evaluate the unknown constants, i.e., $\Delta G_{\mathrm{FePt}}^{\mathrm{A} 1 \rightarrow \mathrm{L}_{0}}, \Delta G_{\mathrm{Al}_{2} \mathrm{O}_{3}}^{\mathrm{am} 1 \rightarrow \mathrm{am} 2}, W_{\mathrm{Al}_{2} \mathrm{O}_{3}-\mathrm{A} 1}$, $\kappa_{\phi}$, and $\kappa_{s}$, which are necessary for calculating the total free energy.

\subsection{Determination of the constants for the energy function}

$\Delta G_{\mathrm{FePt}}^{\mathrm{A} 1 \rightarrow \mathrm{L} 1_{0}}$ is the chemical driving force of ordering from A1 to $\mathrm{L}_{0}$ in the FePt phase. It is calculated using the chemical free energies of the FePt disordered and ordered phases. Recently, Fredriksson and Sundman ${ }^{8)}$ assessed the thermodynamic data for the Fe-Pt binary system based on the CALPHAD method. ${ }^{9)}$ Using their thermodynamic data for chemical free energy, $\Delta G_{\mathrm{FePt}}^{\mathrm{A} 1 \rightarrow \mathrm{L} 1_{0}}$ is calculated as $\Delta G_{\mathrm{FePt}}^{\mathrm{A} 1 \rightarrow \mathrm{L}_{0}}=-2.242 \times 10^{3}\left(\mathrm{~J} \cdot \mathrm{mol}^{-1}\right)$ at $T=923 \mathrm{~K}$ when the composition of FePt phase is $c_{\mathrm{Fe}}=0.55\left(c_{\mathrm{Pt}}=0.45\right)$. $\Delta G_{\mathrm{Al}_{2} \mathrm{O}_{3}}^{\mathrm{am} 1 \rightarrow \mathrm{am} 2}$ is expediently set as $\Delta G_{\mathrm{Al}_{2} \mathrm{O}_{3}}^{\mathrm{am} 1 \rightarrow \mathrm{am} 2}=$ $-\Delta G_{\mathrm{FePt}}^{\mathrm{Al} \rightarrow \mathrm{L}_{0}}$, then a phenomenological requirement that the $\mathrm{a}-\mathrm{Al}_{2} \mathrm{O}_{3}$ phase does not change during ordering of the FePt phase is satisfied automatically.

The value of $W_{\mathrm{Al}_{2} \mathrm{O}_{3}-\mathrm{A} 1}$ is estimated by the morphological microstructure data for the as-sputtered film that was experimentally obtained. ${ }^{6,7)}$ First of all, the two-phase granular structure in the as-sputtered film is considered to be the result of spinodal decomposition on the chemical free energy curve described by the phase-field variables. In the phase-field methodology, since the chemical free energy curves with a different crystal structure are connected to each other using phase-field variables, the mechanism of spinodal decomposition is applicable for all phase separation even if the crystal structure is different. In this case, this phase separation is regarded as the spinodal decomposition from the gaseous solution phase to the two-phase solid state of a$\mathrm{Al}_{2} \mathrm{O}_{3}$ and $\mathrm{FePt}$, and the trajectory of the chemical free energy during the phase decomposition is assumed to be located on the chemical free energy curve of eq. (7). From the spinodal theory, ${ }^{10,11)}$ we have eq. (11).

$$
\begin{aligned}
4 \kappa_{\phi} k_{c}^{2} & =-\frac{\partial^{2} \Delta G_{\mathrm{Al}_{2} \mathrm{O}_{3}-\mathrm{A} 1}}{\partial \phi_{\mathrm{FePt}}^{2}} \\
& =-2 W_{\mathrm{Al}_{2} \mathrm{O}_{3}-\mathrm{A} 1}\left(1-6 \phi_{\mathrm{FePt}}+6 \phi_{\mathrm{FePt}}^{2}\right)
\end{aligned}
$$

The preferential wave number $k_{c}$ is defined by $k_{c} \equiv 2 \pi / \lambda_{c}$, where $\lambda_{c}$ is the preferential wavelength of the spinodal decomposition for the $\phi_{\mathrm{FePt}}$ field. In addition, when the shape of the $\phi_{\mathrm{FePt}}$ profile across the $\mathrm{a}-\mathrm{Al}_{2} \mathrm{O}_{3} / \mathrm{FePt}$ interface possesses a stable profile shape with local equilibrium, eq. (12) is obtained. ${ }^{4,10)}$

$$
\kappa_{\phi}=\frac{9 \gamma_{s}^{2} V_{m}^{2}}{W_{\mathrm{Al}_{2} \mathrm{O}_{3}-\mathrm{A} 1}}
$$

where $V_{m}$ is the molar volume, and $\gamma_{s}$ is the interfacial energy density at the a- $\mathrm{Al}_{2} \mathrm{O}_{3} / \mathrm{FePt}$ interface. Substituting eq. (12) into eq. (11), we get

$$
W_{\mathrm{Al}_{2} \mathrm{O}_{3}-\mathrm{A} 1}=\frac{6 \pi \gamma_{s} V_{m}}{\lambda_{c}} \sqrt{\frac{2}{-\left(1-6 \phi_{\mathrm{FePt}}+6 \phi_{\mathrm{FePt}}^{2}\right)}} .
$$

The experimental value of $\gamma_{s}$ is available ${ }^{12)}$ (see Table 1), and according to the experimental results obtained by Watanabe ${ }^{6}$ et al., $\phi_{\mathrm{FePt}}=0.4$ and $\lambda_{c}=4(\mathrm{~nm})$, so that $W_{\mathrm{Al}_{2} \mathrm{O}_{3}-\mathrm{A} 1}=$ $1.30 \times 10^{5}(\mathrm{~J} / \mathrm{mol})$, and using this, the value of $\kappa_{\phi}$ is calculated from eq. (12). The molar volume of the $\operatorname{FePt}\left(\mathrm{L1}_{0}\right)$ phase was used for $V_{m}$.

The gradient energy coefficient $\kappa_{S}$ is determined by using the experiment data $d_{\mathrm{APB}}$, the width of anti-phase boundary (APB) in the $\mathrm{L}_{0} \mathrm{FePt}$ ordered phase. When the order parameter profile of $s$ across the anti-phase boundary has a stable shape, the condition 
Table 1 Numerical values used for the calculation.

\begin{tabular}{ll}
\hline Aging temperature, $T / \mathrm{K}$ & 923 \\
\hline Composition in FePt phase (atomic fraction) & $\begin{array}{l}c_{\mathrm{Fe}}=0.55, \\
c_{\mathrm{Pt}}=0.45\end{array}$ \\
\hline Chemical driving force, $\Delta G_{\mathrm{FePt}}^{\mathrm{A} 1 \rightarrow \mathrm{L}_{0}} / \mathrm{J} \cdot \mathrm{mol}^{-1}$ & $-2.242 \times 10^{3}$ \\
\hline Interfacial energy density, $\gamma_{s} / \mathrm{J} \cdot \mathrm{m}^{-2}$ & 1.558 \\
\hline Energy barrier between a-Al ${ }_{2} \mathrm{O}_{3}$ and $\mathrm{FePt}$ phases, & $1.30 \times 10^{5}$ \\
$W_{\mathrm{Al}_{2} \mathrm{O}_{3}-\mathrm{Al}} / \mathrm{J} \cdot \mathrm{mol}^{-1}$ & \\
\hline $\mathrm{Gradient}^{-1}$ energy coefficient, $\kappa_{s} / \mathrm{J} \cdot \mathrm{m}^{2} \cdot \mathrm{mol}^{-1}$ & $1.07 \times 10^{-15}$ \\
\hline Molar volume, $V_{m} / \mathrm{m}^{3} \cdot \mathrm{mol}$ & -1 \\
\hline Volume fraction of FePt phase, $\phi_{\mathrm{FePt}}$ & $0.4,0.6$ \\
\hline Wave length of a-Al ${ }_{2} \mathrm{O}_{3}$ and FePt two phases $\mathrm{Volume}$ & 4.0 \\
\hline fraction of FePt phase, $\lambda_{c} / \mathrm{nm}$ & 1.38 \\
\hline Width of APB region, $d_{\mathrm{APB}} / \mathrm{nm}$ & \\
\hline
\end{tabular}

$$
\kappa_{s}=\frac{1}{4} d_{\mathrm{APB}}^{2} \mid \Delta G_{\mathrm{FePt}}^{\mathrm{A} 1 \rightarrow{ }^{\mathrm{L} 1_{0}} \mid}
$$

is satisfied. ${ }^{10)}$ Experimental observations by Watanabe et al. ${ }^{6)}$ indicates that $d_{\mathrm{APB}}=1.38 \times 10^{-9}(\mathrm{~m})$, then the value of $\kappa_{s}$ is $\kappa_{s}=1.07 \times 10^{-15}\left(\mathrm{~J} \cdot \mathrm{m}^{2} / \mathrm{mol}\right)$ for $\mathrm{Fe}-45 \mathrm{at} \% \mathrm{Pt}$ (at $\left.923 \mathrm{~K}\right)$. Numerical values used for the calculation are summarized in Table 1.

Figure 1 is the chemical free energy curve for eq. (9). It is easy to visualize the path of phase decomposition during sputtering and the order-disorder phase transition of FePt phase by using this free energy surface. The initial state of the gaseous solution phase corresponds to the energy level denoted by (A). The path of phase decomposition during sputtering is indicated by the arrows pointing away from (A). The arrow in the direction of (B) corresponds to the path of the order-disorder phase transition in the FePt phase during subsequent heat treatment.

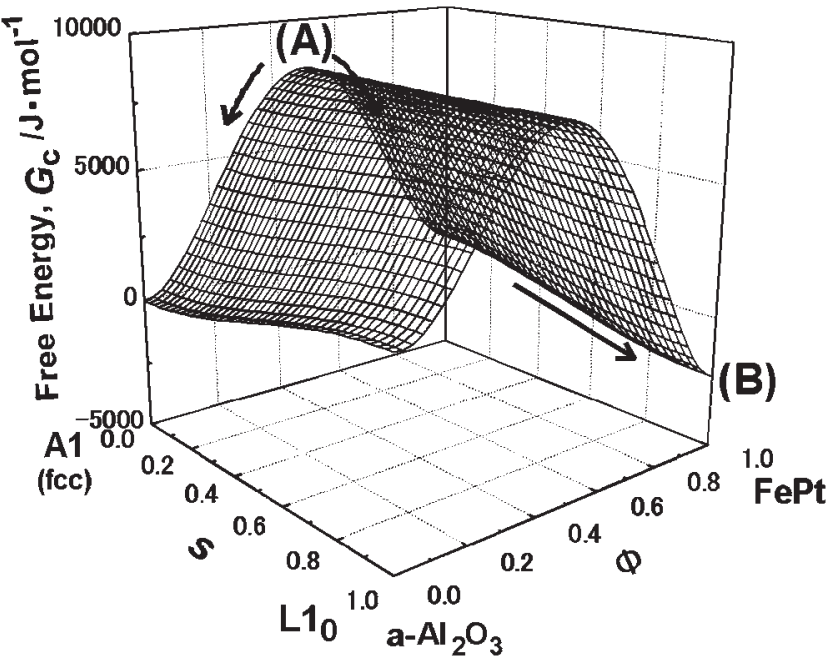

Fig. 1 Chemical free energy of the a- $\mathrm{Al}_{2} \mathrm{O}_{3}-\mathrm{FePt}$ system.

\section{Results of the Phase-Field Simulation}

Figure 2 shows the two-dimensional simulation result for the FePt nano-granular structure formation and the ordering of FePt nano-particles at $923 \mathrm{~K}$. The composition of the FePt phase is set to $\mathrm{Fe}-45$ at\%Pt. The black and white droplet shaped regions are FePt nano-particles. The degree of white refers to the degree of $\mathrm{L}_{0}$ ordering. The black particles are the $\mathrm{FePt}(\mathrm{A} 1)$ disordered phase, and the white particles are the $\mathrm{FePt}\left(\mathrm{L1}_{0}\right)$ ordered phase. And the matrix (gray region) is the $\mathrm{a}-\mathrm{Al}_{2} \mathrm{O}_{3}$ phase. Numerical values in the figure indicate the normalized aging time.

In the initial as-spattered stage, as shown in Figs. 2(a) and (b), FePt phase is in a disordered state. With progress of aging, as seen in Figs. 2(c) to (e), ordering from $\mathrm{A} 1$ to $\mathrm{L}_{0}$

\section{Fe-45at\%Pt at 923K}


Fig. 2 Simulation result of the microstructure changes in FePt nano-granular film at $923 \mathrm{~K}$. Volume fraction of FePt phase is 0.4 . 


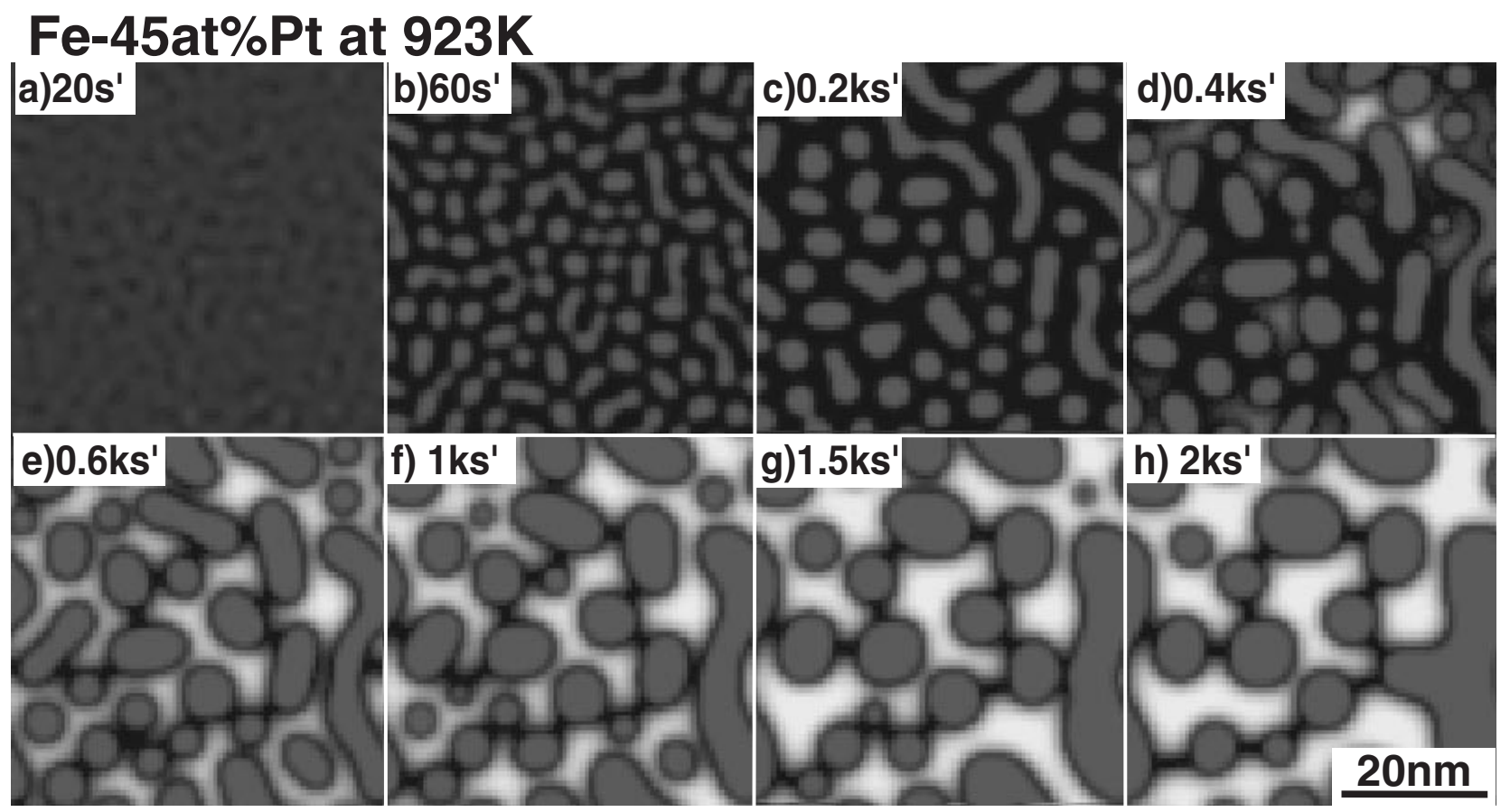

Fig. 3 Simulation result of the microstructure changes in FePt nano-granular film at $923 \mathrm{~K}$. Volume fraction of FePt phase is 0.6.

proceeds and the FePt phase becomes coarser. We observed that the ordering speed of the smaller sized particles was delayed, refer to the left arrow in Fig. 2(e). The anti-phase boundary is also seen in the FePt particle, refer to the right arrow in Fig. 2(e). It is interesting to note that the degree of ordering decreases as the particle size becomes smaller, refer to arrow in Fig. 2(g). This size dependant FePt phase ordering has already been observed experimentally by Takahashi et $a l{ }^{13)}$ These microstructure changes agree well with the FePt nano-granular structure formation experimentally observed. $^{6,7)}$

Since the simulation shown in Fig. 2 is the result of phasefield modeling constructed from experimental data, it is not surprising that the result calculated quantitatively reproduces the experimental microstructure. However, a characteristic of phase-field modeling is that once we construct the phase-field model for a specific case such as in Fig. 2, other microstructure changes are easily calculated without the need for experimentation. The boundary conditions need only be changed slightly. For example, Fig. 3 shows the result for the volume fraction of FePt phase set to $60 \%$. We can see from the figure that the FePt phase forms a matrix when compared with that of Fig. 2. The microstructure change in Fig. 3 shows good agreement with the experimental results by Ping et al. ${ }^{7)}$ Moreover, the calculation time required to finish the simulation took only a few minutes on a standard personal computer (1 GHz CPU or greater). Therefore, it is easy to carry out trial-and-error testing for various microstructure formations on a personal computer by changing the boundary conditions. This modeling method can also be applied to various types of granular structural formations.

Finally we would like to emphasize that the microstructure's morphological shape is quantitatively obtained using phase-field modeling, therefore, it is also possible to calculate magnetic properties, i.e., magnetic hysteresis, based on the micromagnetics ${ }^{14)}$ simulation by using the calculated microstructure data as boundary conditions.

\section{Conclusions}

We attempted to model the complex microstructural changes found in FePt nano-granular structure formation and the order-disorder phase transition of FePt nano-particle using the phase-field method. The results obtained are as follows:

(1) We quantitatively modeled FePt nano-granular structure formation using the phase-field method.

(2) This method could be applied to the modeling of various types of granular structure formations.

(3) The simulation results suggested that FePt nano-particle ordering is size dependent.

(4) The modeling of microstructure evolution using the phase-filed modeling, is an effective strategy in predicting and analyzing complex microstructure formations.

\section{Acknowledgements}

The authors are grateful to Dr. Y. K. Takahashi for her helpful discussions. This work was partly supported by a NEDO International Joint Research Grant on "Structuring Knowledge, Science and Technology for Nano Material Processing", and by a Special Coordination Fund for Promoting Science and Technology on "Nanohetero Metallic Materials" from the Ministry of Education, Culture, Sports, Science and Technology. 


\section{REFERENCES}

1) T. Koyama: Materia Japan 42 (2003) 397-404.

2) T. Koyama, T. Kozakai and T. Miyazaki: Materia Japan 38 (1999) 624 628

3) L.-Q. Chen: Annu. Rev. Mater. Res. 32 (2002) 113-40.

4) W. J. Boettinger, J. A. Warren, C. Beckermann and A. Karma: Annu. Rev. Mater. Res. 32 (2002) 163-94.

5) M. Ode, S. G. Kim and T. Suzuki: ISIJ Int. 41 (2001) 1076-82.

6) M. Watanabe, T. Masumoto, D. H. Ping and K. Hono: Appl. Phys. Lett. 76 (2000) 3971-3973.

7) D. H. Ping, M. Ohnuma, K. Hono, M. Watanabe, T. Iwasa and T. Masumoto: J. Appl. Phys. 90 (2001) 4708-16.
8) P. Fredriksson and B. Sundman: CALPHAD 25 (2001) 535-548.

9) N. Saunders and A. P. Miodownik: CALPHAD, (Pergamon, 1998).

10) J. W. Cahn: The Selected Works of J. W. Cahn, ed. by W. C. Carter and W. C. Johnaon, (TMS, 1998) pp. 29-50.

11) J. E. Hilliard: Phase Transformation, ed. by H. I. Aaronson, (ASM, Metals Park, Ohio, 1970) pp. 497-560.

12) L. E. Murr: Interfacial Phenomena in Metals and Alloys, (AddisonWesley, 1975) pp. 155.

13) Y. K. Takahashi, M. Ohnuma and K. Hono: Collected Abstracts of the 2002 Autumn Meeting of the Japan Inst. Metals, (2002) 64.

14) D. V. Berkov, K. Ramstock and A. Hubert: Phys. Status. Solidi. 137 (1993) 207-25. 